\title{
Quelles sont les conséquences socio-économiques des exacerbations de BPCO ? 资
}

\section{What are the socio-economic consequences of acute COPD exacerbations?}

\author{
A. Bourdin, N. Molinari* \\ Inserm U1046, département de pneumologie et addictologie, CHU de Montpellier, \\ 34070 Montpellier, France
}

\section{Introduction}

Les exacerbations de BPCO (EABPCO) représentent des coûts directs et indirects difficiles à apprécier et extrêmement variables selon les études et les pays [1,2]. La sévérité des exacerbations est un critère majeur affectant leurs coûts, un séjour en réanimation notamment. A contrario, les exacerbations non reportées (et donc sans traitement ou sans ajout de nouveau traitement [EABPCO légère]) sont assez mal connues et leur coût direct nul ou quasi nul devrait affecter les coûts moyens mais dans une proportion impossible à apprécier. D'autre part, le sous diagnostic et le poids des comorbidités compliquent ces estimations [3].

Il est maintenant possible d'évaluer non plus uniquement les coûts directs liés à une pathologie, mais l'excès de coût [4]. En effet, il est nécessaire de considérer qu'une personne d'un âge donné «en bonne santé apparente » est génératrice d'un coût pour le système de santé, et il est donc plus juste d'évaluer non pas les coûts directs de la BPCO mais les excès de coûts eu égard à cette référence. Si cela est actuellement possible pour évaluer l'excès de coût sur une année par exemple de la pathologie dans son ensemble, cela reste à ce jour inconnu pour les seules exacerbations.

\footnotetext{
* Auteur correspondant. Inserm U1046, département de l'information médicale, hôpital La Colombière, CHU de Montpellier, 34070 Montpellier, France.

Adresse e-mail : nicolas.molinari@inserm.fr (N. Molinari).
} 


\section{Coûts des exacerbations}

\section{Coûts des exacerbations non hospitalisées}

Peu d'études sont disponibles dans ce domaine (Tableau 1).

On peut estimer que les exacerbations définies uniquement par une augmentation des symptômes enregistrées dans les études randomisées contrôlées représentent les exacerbations non rapportées et expliquent donc les coûts directs faibles associés. Dans l'ensemble les études rapportent rarement le détail des coûts, et jamais les coûts indirects (par exemple l'absentéisme professionnel). La plupart rapportent également le coût des exacerbations hospitalisées alors qualifiées de sévères. $\mathrm{Si}$ d'importantes variations apparaissent entre les différentes études menées dans les différents pays selon différentes méthodes, on retiendra un prix moyen oscillant entre 15 et 300 euros/exacerbation non hospitalisée, le prix des médicaments étant la part fixe et le recours aux soins la part variable (recours à un service d'urgence, recours à de la kinésithérapie respiratoire ou à des nébulisations).

\section{Coût des exacerbations hospitalisées}

Dans l'ensemble ces données sont beaucoup plus simples à obtenir, les coûts indirects par contre n'étant en général qu'assez mal connus (Tableau 1). Les coûts moyens sont de l'ordre de 4500-5000 USD (3500-4000 euros) mais peu d'études ont étudié le prix des admissions en réanimation par exemple. Ces exacerbations représentent des coûts incompressibles directs dix à cent fois supérieurs aux exacerbations non hospitalisées même si des prises en charge à domicile commencent à émerger. De très grandes variations sont notées selon les sources de données, les frais pris en compte (surcoût par rapport à des gens du même âge par exemple, ou date index le jour de l'admission jusqu'à 30 jours). Cette variation peut aussi être due à la différence entre les différents systèmes de santé. À la différence des États-Unis, nous sommes à la T2A en France avec des « forfaits » par séjour, quels que soient les actes réalisés.

Les sources de coûts sont assez bien détaillées dans certains travaux. La part médicamenteuse est très faible en général, écrasée par les frais des soins (personnel, examens, transport qui dépassent les $70 \%$ du coût total) [5] en particulier en soins intensifs. En France on estime qu'il y a plus de 130000 admissions en hospitalisation complète (avec à minima une nuit) par an pour exacerbation de BPCO, en augmentation de $15,5 \%$ entre 2007 et 2012. La mortalité intrahospitalière des exacerbations de BPCO en France en 2012 atteignait 7,9 \% Le coût annuel total direct en 2012 a avoisiné les 680 millions d'euros [6]. Les coûts indirects ou intangibles sont particulièrement difficiles à apprécier (qualité de vie réduite, présence d'un accompagnant qui lui aussi perd du temps pour accompagner, manque de productivité au travail) («presenteeism » des anglosaxons, qui désigne la présence au travail mais dans des conditions de santé telles que la qualité du travail est impacté, absentéisme, handicap, alitement, jours d'activité restreinte) $[7,8]$. On peut estimer qu'ils sont importants dans cette pathologie, même si l'absentéisme professionnel reste limité par la classe d'âge concernée. Très peu de publications originales ont réellement étudié en détails cette question dans le cadre restreint des exacerbations. Britton en 2003 [9] estimait pour le Royaume-Uni un coût sociétal additionnel d'environ $15 \%$ (faisant passer l'enveloppe globale de 1255 à 1453 millions d'euros annuels dépensés pour la BPCO). Aux États-Unis, l'absentéisme lié à la BPCO chez l'adulte est estimé à 3,9 milliards de dollars (vs. un coût total lié à

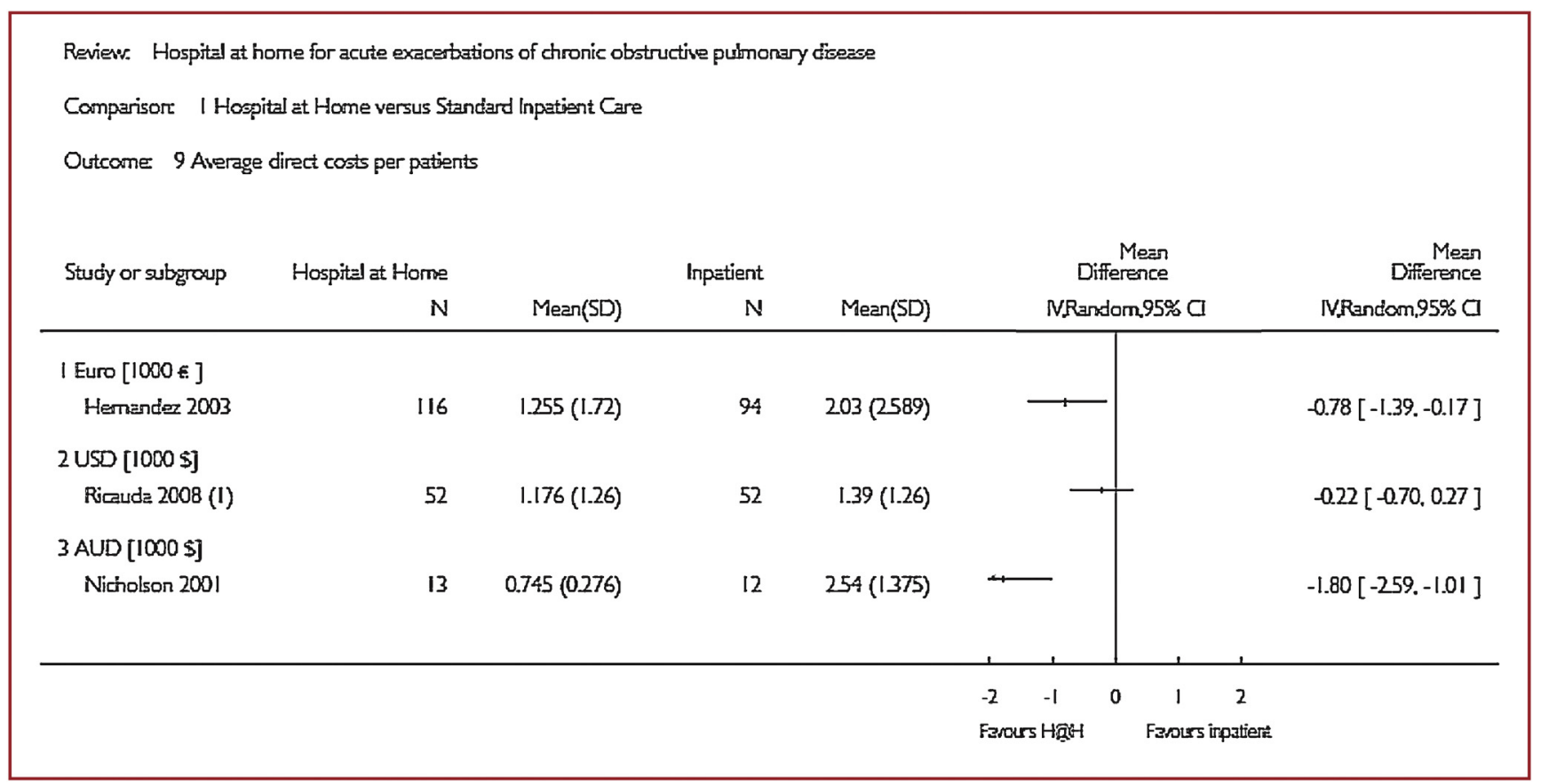

Figure 1. Reproduction en diagramme de Forest des trois études comparatives de la prise en charge en hospitalisation à domicile (HAD) vs hospitalisation conventionnelle pour les exacerbations de bronchopneumopathie chronique obstructive (BPCO) sans signe de gravité initiale [12]. 
Tableau 1 Principales études estimant le coût des exacerbations de BPCO (hospitalisées en gras).

\begin{tabular}{|c|c|c|c|c|c|c|}
\hline Étude & Pays & Design & $\begin{array}{l}\text { Nombre de } \\
\text { patients }\end{array}$ & $\begin{array}{l}\text { Définition des } \\
\text { exacerbations }\end{array}$ & $\begin{array}{l}\text { Coûts directs des } \\
\text { exacerbations }\end{array}$ & $\begin{array}{l}\text { Détails } \\
\text { des coûts }\end{array}$ \\
\hline Price, 1999 & $\begin{array}{l}\text { World } \\
\text { Monde mais } \\
\text { seulement } 194 \text { EA }\end{array}$ & Essai RCT (flutisone) & 194 & Traitement & $\begin{array}{l}\text { Légère } 39 \$ \\
\text { Modérée } 248 \$ \\
\text { Sévères (hospit) } 4313 \$\end{array}$ & Non \\
\hline Miravitlles et al., 2002 [13] & Espagne & Consultations MG & 2414 & Oui (symptômes) & $260 \$$ & Non \\
\hline Andersson et al., 2002 [14] & Suède & $\begin{array}{l}\text { Rétrospectif fréquent } \\
\text { exacerbateur }\end{array}$ & 61 & $\begin{array}{l}\text { Oui (recours aux } \\
\text { soins) }\end{array}$ & $\begin{array}{l}23-68 \$ \\
403 \$ \\
4172 \$\end{array}$ & Non \\
\hline Oostenbrink et al., 2004 [15] & Hollande/Belgique & RCT (tiotropium) & 519 & Oui (symptômes) & $\begin{array}{l}150 \$ \\
1011 \$ \\
6996 \$\end{array}$ & Oui \\
\hline Lucioni, 2005 & Italie & Suivi & 570 & Non & $1751 \$$ & Non \\
\hline O’Reilly et al., 2006, [16] & UK & $\mathrm{RCT}$ & 848 & $\begin{array}{l}\text { Oui (symptômes et } \\
\text { ressources) }\end{array}$ & $\begin{array}{l}29 \$ \\
55 \$\end{array}$ & Non \\
\hline Rutten-van Molken et al., 2007 [17] & Espagne & $\begin{array}{l}\text { Analyse post-hoc } \\
\text { Miravitlles, } 2002\end{array}$ & 2414 & $\begin{array}{l}\text { Partielle (définition } \\
\text { de sévérité ?) }\end{array}$ & $\begin{array}{l}\text { Non sévères } 121 \$ \\
\text { Sévères } 3132 \$\end{array}$ & Oui \\
\hline O’Reilly et al., 2007 [18] & UK & Prospectif 1 centre & 149 & Oui (hospitalisation) & $4659 \$$ & Oui \\
\hline Simoens et al., 2007 [19] & Belgique & Rétrospectif 1 centre & 267 & Oui (hospitalisation) & 7757\$ & Non \\
\hline Mittmann et al., 2008 [20] & Canadienne & $\begin{array}{l}\text { Observationnel } \\
\text { prospectif }\end{array}$ & 609 & Oui & $\begin{array}{l}\text { Légère } 120 \$ \\
\text { Modérée } 608 \$ \\
\text { Sévères (hospit) } 9060 \$\end{array}$ & Oui \\
\hline Blasi et al., 2014 [5] & $\begin{array}{l}\text { Lombardie } \\
\text { (Italie) }\end{array}$ & PMSI & 15857 & $\begin{array}{l}\text { Oui (hospitalisation } \\
\text { avec antibiotique ou } \\
\text { corticoïdes) }\end{array}$ & $6725 €$ & Non \\
\hline Kim et al., 2013 [21] & Corée Sud & PMSI & 174106 & Hospitalisés & 2580 US\$ & Partiel \\
\hline Miratvitlles et al., 2013 [22] & Espagne & Consultations MG & 260 & Ambulatoires & $344,96 €$ & Oui \\
\hline Abudagga et al., 2013 [23] & US & PMSI branche de santé & 17382 & $\begin{array}{l}\text { Traitement } \\
\text { médicamenteux }\end{array}$ & $\begin{array}{l}\text { Non hospitalisés } \\
\$ 269 \pm \$ 748 \\
\text { Hospitalisés } \\
\$ 18,120 \pm \$ 31,592\end{array}$ & Non \\
\hline de Miguel-Diez et al., 2013 [24] & Espagne & PMSI national & 215835 & Hospitalisés & 4129 euros & Non \\
\hline Ornek T. Int J Med Sci 2012 & Turquie & Suivi prospectif & 242 & Hospitalisés & $\$ 1765 \pm 2139$ & Oui \\
\hline Ozkaya et al., 2011 [25] & Turquie & Rétrospectif 1 centre & 7832 & Hospitalisés & US\$718 \pm 364 & Oui \\
\hline Yu et al., 2011 [26] & US & $\begin{array}{l}\text { Base de données } \\
\text { d'Assurance }\end{array}$ & 228978 & Oui & 3439 US\$ & Non \\
\hline Geitona et al., 2011 [27] & Grèce & Rétrospectif 1 centre & 142 & Hospitalisés & $1711 €$ & Oui \\
\hline Dalal et al., 2011 [28] & US & $\begin{array}{l}\text { Admissions via urgences } \\
\text { de } 602 \text { hôpitaux }\end{array}$ & 71493 & Hospitalisés & $5754 \$$ & Oui \\
\hline
\end{tabular}




\begin{tabular}{|c|c|c|c|c|c|c|c|}
\hline Étude & Année & $\begin{array}{l}\text { Nombre de } \\
\text { jours par an } \\
\text { d'absence }\end{array}$ & «Présentéisme» & $\begin{array}{l}\text { Handicap } \\
\text { temporaire } \\
\text { (jours) }\end{array}$ & $\begin{array}{l}\text { Handicap } \\
\text { long terme } \\
\text { (jours) }\end{array}$ & $\begin{array}{l}\text { Jours } \\
\text { activité } \\
\text { restreinte }\end{array}$ & $\begin{array}{l}\text { Jours } \\
\text { d'alitement }\end{array}$ \\
\hline $\begin{array}{c}\text { Strassels et al., } \\
2001 \text { [29] }\end{array}$ & 1987 & 1 & & & & 15,9 & 16,1 \\
\hline Ward, 2002 & 1993-1994 & 1,5 & & & & & \\
\hline Mannino, 2002 & 1996 & 2,4 & & & & 31 & 13,1 \\
\hline Halpern, 2003 & 2000 & 1,7 & & & & & \\
\hline Nair, 2012 & $2000-2007$ & $0,3 \mathrm{~h} / \mathrm{mois}$ & & $0,3 \mathrm{j} / \mathrm{mois}$ & & & \\
\hline $\begin{array}{l}\text { DiBonaventura, } \\
2012\end{array}$ & 2009 & $1,88 \mathrm{~h} / \mathrm{sem}$ & $4,82 \mathrm{~h} / \mathrm{sem}$ & & & & \\
\hline Wang, 2003 & 2000 & $\begin{array}{l}\text { 19,4 (excès lié } \\
\text { à la BPCO) }\end{array}$ & 27,5 & & & & \\
\hline Darkow, 2007 & 2001-2004 & & & 54,8 & 76,4 & & \\
\hline
\end{tabular}

la BPCO de 32,1 milliards, soit une fois encore proche de $15 \%$ [ [4]. Les auteurs spécifient cependant que cet absentéisme ne peut être lié avec certitude avec les exacerbations [10]. Le Tableau 2 reprend les principales données socioéconomiques des coûts indirects [11].

\section{Modulation des conséquences socio-économiques}

Très peu de stratégies ont réellement pu évaluer ce point. Cependant, la prise en charge en hospitalisation à domicile (HAD) comme alternative à l'hospitalisation conventionnelle dans les EABPCO sans signe de gravité initiale a pu faire l'objet d'une méta-analyse. Elle reste hélas relativement limitée car trois études seulement (dont aucune n'a été effectuée en France) ont pu être cumulées et des effectifs trop faibles ne permettent pas la méta-analyse. Le résultat indique - au-delà de la supériorité clinique abordée dans le chapitre dédié - une tendance favorable en termes de coûts socio-économiques (Fig. 1) [12]. L'organisation du système français actuel de santé ne permet pas de recommander l'HAD pour la prise en charge des EABPCO (G2C).

\section{Financement}

L'actualisation des recommandations sur la BPCO est financée par la SPLF, promoteur de l'opération, sur ses fonds propres.

\section{Déclaration de liens d'intérêts}

Tous les membres du Comité d'organisation, du Groupe de travail et du Groupe de lecture ont renseigné un formulaire de déclaration d'intérêts. Ces déclarations sont publiques et disponibles au siège de la SPLF.

\section{Références}

[1] Toy EL, Gallagher KF, Stanley EL, et al. The economic impact of exacerbations of chronic obstructive pulmonary disease and exacerbation definition: a review. COPD 2010;7:214-28.

[2] Chapman KR, Mannino DM, Soriano JB, et al. Epidemiology and costs of chronic obstructive pulmonary disease. Eur Respir J 2006;27:188-207.

[3] Pauwels RA, Rabe KF. Burden and clinical features of chronic obstructive pulmonary disease (COPD). Lancet 2004;364:613-20.

[4] Mannino DM, Buist AS. Global burden of COPD: risk factors, prevalence, and future trends. Lancet 2007;370:765-73.

[5] Blasi F, Cesana G, Conti S, et al. The clinical and economic impact of exacerbations of chronic obstructive pulmonary disease: a cohort of hospitalized patients. PLoS One 2014;9:e101228.

[6] Bourdin A, Chanez P, Marin G, et al. Exhaustive analysis of admissions for COPD exacerbation in France 2007-2012. In: Congrès de Pneumologie de Langue Française. 2015.

[7] DiBonaventura M, Paulose-Ram R, Su J, et al. The burden of chronic obstructive pulmonary disease among employed adults. Int J Chron Obstruct Pulmon Dis 2012;7:211-9.

[8] DiBonaventura M, Paulose-Ram R, Su J, et al. The impact of COPD on quality of life, productivity loss, and resource use among the elderly United States workforce. COPD 2012;9:46-57.

[9] Britton M. The burden of COPD in the UK: results from the Confronting COPD survey. Respir Med 2003;97:S71-9.

[10] Ford ES. Hospital discharges, readmissions, and emergency department visits for chronic obstructive pulmonary disease or bronchiectasis among US adults: findings from the Nationwide Inpatient Sample 2001-2012 and Nationwide Emergency Department Sample 2006-2011. Chest 2015;147:989-98.

[11] Patel JG, Nagar SP, Dalal AA. Indirect costs in chronic obstructive pulmonary disease: a review of the economic burden on employers and individuals in the United States. Int J Chron Obstruct Pulmon Dis 2014;9:289-300.

[12] Jeppesen E, Brurberg KG, Vist GE, et al. Hospital at home for acute exacerbations of chronic obstructive pulmonary disease. Cochrane Database Syst Rev 2012;5:CD003573. 
[13] Miravitlles M, Murio C, Guerrero T, et al. Pharmacoeconomic evaluation of acute exacerbations of chronic bronchitis and COPD. Chest 2002;121:1449-55.

[14] Andersson F, Borg S, Jansson SA, et al. The costs of exacerbations in chronic obstructive pulmonary disease (COPD). Respir Med 2002;96:700-8.

[15] Oostenbrink JB, Rutten-van Molken MP, Al MJ, et al. Oneyear cost-effectiveness of tiotropium versus ipratropium to treat chronic obstructive pulmonary disease. Eur Respir J 2004;23:241-9.

[16] O’Reilly JF, Williams AE, Holt K, et al. Defining COPD exacerbations: impact on estimation of incidence and burden in primary care. Prim Care Respir J 2006;15:346-53.

[17] Rutten-van Molken MP, Oostenbrink JB, Miravitlles M, et al. Modelling the 5-year cost-effectiveness of tiotropium, salmeterol and ipratropium for the treatment of chronic obstructive pulmonary disease in Spain. Eur J Health Econ 2007;8: $123-35$.

[18] O'Reilly JF, Williams AE, Rice L. Health status impairment and costs associated with COPD exacerbation managed in hospital. Int J Clin Pract 2007;61:1112-20.

[19] Simoens S, Decramer M, De Coster S, et al. Clinical and economic analysis of antimicrobial therapy of chronic obstructive pulmonary disease exacerbations. Int J Clin Pract 2007;61:200-6.

[20] Mittmann N, Kuramoto L, Seung SJ, et al. The cost of moderate and severe COPD exacerbations to the Canadian healthcare system. Respir Med 2008;102:413-21.
[21] Kim J, Rhee CK, Yoo KH, et al. The health care burden of high grade chronic obstructive pulmonary disease in Korea: analysis of the Korean Health Insurance Review and Assessment Service data. Int J Chron Obstruct Pulmon Dis 2013;8:561-8.

[22] Miravitlles M, Garcia-Polo C, Domenech A, et al. Clinical outcomes and cost analysis of exacerbations in chronic obstructive pulmonary disease. Lung 2013;191:523-30.

[23] Abudagga A, Sun SX, Tan H, et al. Exacerbations among chronic bronchitis patients treated with maintenance medications from a US managed care population: an administrative claims data analysis. Int J Chron Obstruct Pulmon Dis 2013;8:175-85.

[24] de Miguel-Diez J, Jimenez-Garcia R, Hernandez-Barrera V, et al. Trends in hospital admissions for acute exacerbation of COPD in Spain from 2006 to 2010. Respir Med 2013;107:717-23.

[25] Ozkaya S, Findik S, Atici AG. The costs of hospitalization in patients with acute exacerbation of chronic obstructive pulmonary disease. Clinicoecon Outcomes Res 2011;3:15-8.

[26] Yu AP, Yang H, Wu EQ, et al. Incremental third-party costs associated with COPD exacerbations: a retrospective claims analysis. J Med Econ 2011;14:315-23.

[27] Geitona M, Hatzikou M, Steiropoulos P, et al. The cost of COPD exacerbations: a university hospital-based study in Greece. Respir Med 2011;105:402-9.

[28] Dalal AA, Shah M, D'Souza AO, et al. Costs of COPD exacerbations in the emergency department and inpatient setting. Respir Med 2011;105:454-60.

[29] Strassels SA, Smith DH, Sullivan SD, et al. The costs of treating COPD in the United States. Chest 2001;119:344-52. 